\title{
Compensation of Unidirectional Geometric Distortion in EPI Using Spline Warping
}

\author{
Jan Kybic \\ Philippe Thévenaz \\ Michael Unser \\ Jan.Kybic@epfl.ch Philippe.Thevenaz@epfl.ch Michael.Unser@epfl.ch \\ Biomedical Imaging Group, DMT/IOA \\ Swiss Federal Institute of Technology Lausanne \\ CH-1015 Lausanne EPFL, Switzerland
}

\begin{abstract}
Due to magnetic field inhomogeneities, EPI images are geometrically distorted, predominantly along the phase-encoding direction. Currently, the distortion is either ignored or compensated manually using a warping function defined through a set of landmarks. We propose an automatic method to unwarp the geometric distortion of EPI images by registering them with corresponding undistorted anatomical MRI images. We show that cubic splines are optimal interpolating functions for both landmark interpolation and approximation. We will consequently use the same warping space in our algorithm which replaces landmarks by an image difference criterion. B-splines are used as generating functions, which leads to a fast and accurate computation. Multiresolution gives robustness and additional speedup. The algorithm performance was evaluated using both real and synthetic data and was found superior to the manual method.
\end{abstract}

\section{Introduction}

Echo planar imaging (EPI) [1] is a fast magnetic resonance imaging technique. It permits an acquisition of a two-dimensional slice using a single excitation, which leads to very short scan times. It is used mainly for functional magnetic resonance imaging (fMRI), the in vivo non-invasive study of the temporal, spatial, and behavioral dependencies of brain activities. In contrast to conventional MRI, where the number of excitations per slice is equal to the number of scan lines, in EPI, the magnetic field gradients encodes two coordinates simultaneously. As one of the gradients (the so called phase-encoding gradient) is several orders of magnitude weaker than the other, the inhomogeneous magnetic field (due mainly to varying magnetic susceptibility of the subject) will manifest itself mainly as a geometric distortion along the direc- tion of the phase-encoding gradient. Symbolically,

$$
f^{o}(g(x, y), y) \simeq f^{i}(x, y)
$$

where $f^{o}$ is the observed EP image, $f^{i}$ is the hypothetical ideal undistorted EP image and $g$ is the unknown warping function.

In order to enable meaningful postprocessing of fMRI images, as well as accurate localization of the activation centers, the distortion must be compensated for. To recover $g$, we register $f^{o}$ with a corresponding undistorted anatomical MRI image $f^{a}$. Knowing $g$, the observed EPI image $f^{o}$ can be unwarped to get (an approximation of) the ideal EPI image $f^{i}$. Manual registration is a tedious and time consuming process that requires an expert; its precision is inherently limited. Due to a large number of scans per examination, the manual registration is impractical and is often skipped. Hence there is an interest for an automatic method.

\section{Related work}

To the best of our knowledge, no other algorithm solves this particular task. Neither rigid-body transform methods [2], nor fluid transform methods [3], nor harmonic function based methods [4] search the desired warping function space. Landmark-based methods [5] lack robustness.

\section{Warp space}

The manual method requires an expert to define a set of corresponding point pairs $\left(\mathbf{x}_{i}, \mathbf{y}_{i}\right)$. The deformation function $g$ is found to pass directly through these landmarks and is interpolated between them. Currently this is done by the program xmorph [6] or our proprietary code which implements the thin-plate spline warping of Bookstein [7].

The common point between the manual method and the automatic algorithm we have developed is the 
cubic spline warp space. In this section, we want to motivate this choice. We show that this model is optimal in a well-defined sense.

For the sake of simplicity, we consider only the $1 \mathrm{D}$ problem here; i.e., the task of recovering an univariate warping function $g: \mathrm{R} \rightarrow \mathrm{R}$. First, it can be shown that a function $g(x)$ satisfying $g\left(x_{i}\right)=y_{i}$ and constrained to minimize $\int\left|g^{\prime \prime}(x)\right|^{2} \mathrm{~d} x$ (minimum curvature solution) is a cubic spline [8]. This situation corresponds to $g$ passing exactly through landmarks. Second, as it is often difficult to obtain precise landmarks, we consider a least-squares fit. We find that a function $g$ minimizing $\sum_{i}\left(f\left(x_{i}\right)-y_{i}\right)^{2}$ under an a priori constraint $\int\left|g^{\prime \prime}(x)\right|^{2} \mathrm{~d} x \leq \alpha$ is also a cubic spline [9]. In both cases, the warping function $g$ can be expressed as a linear combination of B-splines, which have a compact support. This leads to a fast algorithm. The argument generalizes to multiple dimensions for landmarks on a cartesian grid.

\section{Automatic Registration}

We want to imitate the manual method while improving the speed and precision of the process. The algorithm flow is depicted in Figure 1. The anatomical, resp. EPI input images are first preprocessed to obtain reference $\left(f^{r}\right.$ from $\left.f^{a}\right)$, resp. test $\left(f^{t}\right.$ from $\left.f^{o}\right)$ images with similar characteristics. Instead of facing the difficult task of landmark detection, we minimize the mean-square error between the reference and warped test images $E=\left\|f_{r}(x, y)-f_{t}(g(x, y), y)\right\|_{l_{2}}^{2}$.

Following the above argument, we explore the same solution space as in the manual case (cubic splines), using the same a priori constraint $\int\left|g^{\prime \prime}\right|^{2} \mathrm{~d} x \leq \alpha$. However, since there are now no explicit landmarks available to put the knots on, we will distribute the knots uniformly over the image. Thus, the warping function is also modeled using cubic B-splines as

$$
g(x, y)=\sum c_{k l} \beta\left(x / h_{x}-k\right) \beta\left(y / h_{y}-l\right)
$$

where $h_{x}$ and $h_{y}$ determine the spacing between knots of the uniform grid and $c_{k l}$ are the parameters associated with each knot. Thanks to good approximation properties of cubic B-splines, we can characterize typical warping function using a small set of parameters $c_{k l}$ with a small error. The knot spacing controls the number of parameters and adjusts the flexibility of the warp. This model is very general while comprising linear warping as a special case.

Multiresolution is used twice in our algorithm: a multiresolution of images and a multiresolution of the deformation model. The registration process starts at the coarsest level and the results are progressively refined using higher-resolution images and finer

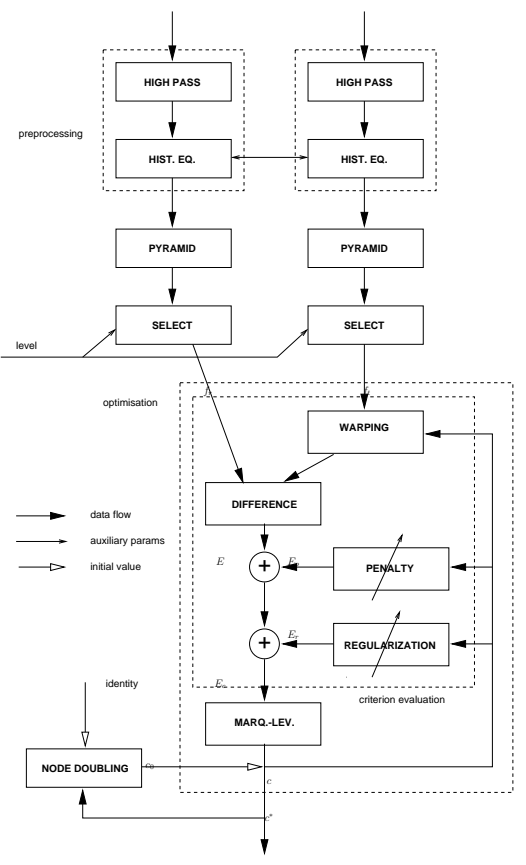

Figure 1: Algorithm overview

control grids until the target resolution is reached. The spline model leads to simple and exact transitions between resolution levels.

Like for the warping function, we interpolate the test image $f_{t}$ using a cubic spline model [10, 11], in order to obtain high accuracy. This is especially important at coarse levels of the image pyramid, because it permits to obtain a very good estimate of the solution, which then acts as a good starting guess for the optimization at the next finer level. The image pyramid is created by least-squares projection using the same paradigm; this consistency minimizes the approximation error.

Optimizing the registration criterion using a Lagrange multiplier method, the augmented cost function becomes $E_{c}=\left\|f_{r}(x, y)-f_{t}(g(x, y), y)\right\|_{l_{2}}^{2}+$ $\lambda\left\|g^{\prime \prime}\right\|_{L_{2}}^{2}$. The measure $E_{c}$ is minimized with respect to the coefficients $c$ using a regularized version of the Newton method, inspired by the MarquardtLevenberg algorithm.

\section{Results}

The algorithm accuracy was tested on several hundreds of test images. We also worked with one full set of brain slices, consisting of thirty anatomical and EPI image pairs. Furthermore, we took the anatomical images from this set and modified them so that they resemble as much as possible real EPI images. These artificial EPI images, deformed by a known 
warping, as well as the real EPI images, were manually registered with corresponding anatomical images by three different people, including one experienced practitioner, for the purpose of comparison with the automatic method.

First of all, anatomical MRI images were deformed by randomly generated transformations from the representable class. A warping index $\varpi$ as defined in [12] (mean of $\left\|g(x, y)-g^{*}(x, y)\right\|$ in the region of interest) is used to characterize the precision of the registration. Under ideal conditions, if the images contain sufficient level of detail, we can achieve a precision up to $\varpi \simeq 10^{-6}$ pixels.

In the same setting, we varied the registration parameters. Figure 2 displays the resulting $E$ and $\varpi$ as a function of the degree $n$ of the splines used to represent $g$ and the knot spacing $h$. It clearly shows the benefit of using a high degree $n$ in high-precision applications. The minimum achievable error $\varpi_{\min }$ (computed by projecting the known warping function on the available solution space) and the associated SSD is shown by the dotted line for the cubic case and marked optimal; note that, except for the smallest knot spacing $h$, it is very close to the calculated values. Furthermore, the similarity of behavior of the criterion $E$ and the true error confirms the suitability of the criterion.

Figure 3 demonstrates the dependence of the registration accuracy on the signal-to-noise ratio. For this series of experiments, the test images were obtained from a known transformation of a reference image with various levels of added white Gaussian noise. An inspace (i.e., representable) deformation was used.

The next series of experiments deals with artificial EPI images, in order to compare the precision and speed of the automatic and manual methods. By filtering and performing histogram modification of the thirty reference anatomical MRI images, we obtain images that are visually equivalent to the corresponding EPI images (Figure 4). After having warped them with a known randomly generated deformation, we use them as test images. The resulting reference/test image pairs were registered automatically as well as manually. Results are summarized in Table 1, comparing the warping index $\varpi$ and the error $E$ for images before and after the registration, using the known true warping and the projection of the warping onto the warp space.

Finally, the algorithm was also tested on real data. Figures 6 and 7 show a typical EPI image before and after registration and unwarping. Contours of the anatomical image (Figure 5) are superimposed over
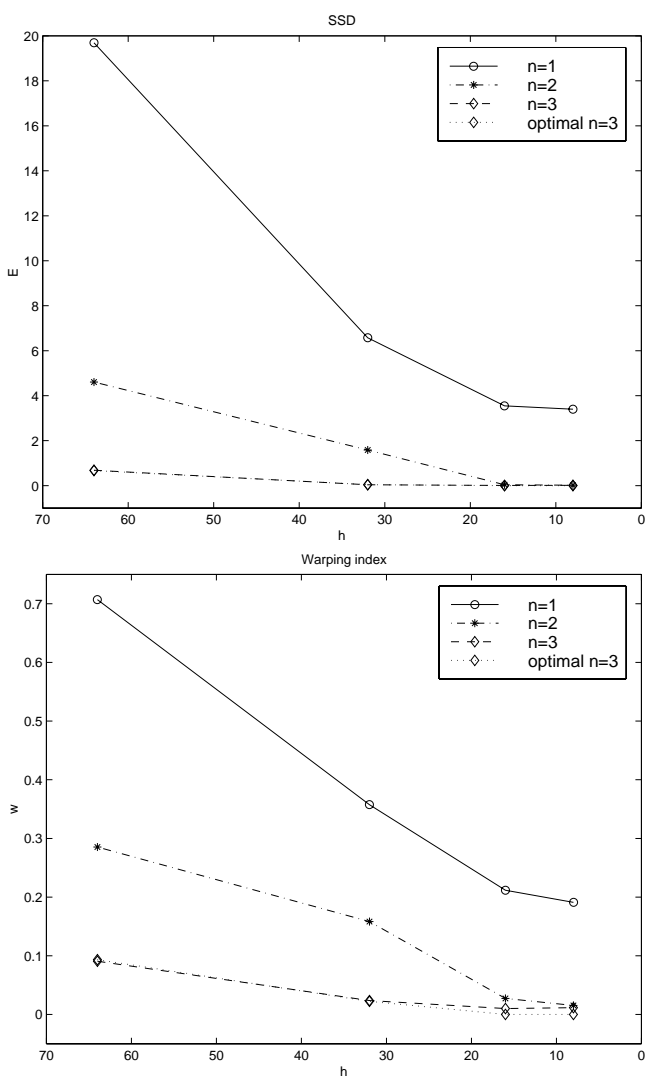

Figure 2: The quality of the registration as a function of the warp spline degree and the knot spacing. The initial values (prior to the registration) were $E=90.9$ and $\varpi=4.3$. Each point shown represents the average of thirty experiments.

the images. For comparison, manual registration results are shown in Figure 8.

\section{Conclusions}

We presented an algorithm for an important problem in fMRI imaging, which so far had been ignored or had to be solved manually.

The algorithm is readily generalizable to more image dimensions, as well as more warping function dimensions, and should therefore be applicable to a variety of other image registration problems where a general non-linear warping function is sought.

\section{References}

[1] Z.-H. Cho, J. P. Jones, and M. Singh, Foundations of Medical Imaging. John Wiley \& Sons, 1993.

[2] J. West et al., "Comparison and evaluation of retrospective intermodality brain image registration 


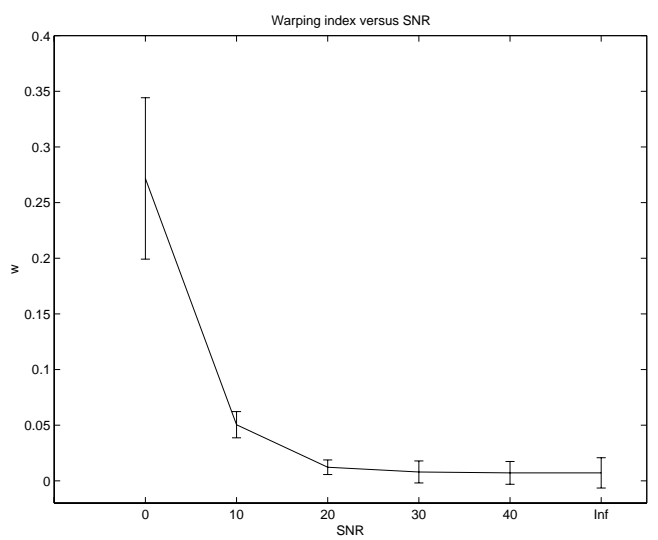

Figure 3: The quality of the registration as a function of the SNR in dB. $n=2, M=6, \varpi_{\text {before }}=3.93$. The error bars mark one standard deviation. Thirty registration experiments per point were performed.

\begin{tabular}{|c||r|r|r|r|r|}
\hline & before & manual & automatic & ideal & true \\
\hline$\varpi$ & 2.8 & 2.6 & 0.5 & 0.1 & 0 \\
\hline$E$ & 231 & 89 & 63 & 71 & 64 \\
\hline time & & $\sim 5 \mathrm{~min}$ & $\sim 30 \mathrm{~s}$ & & \\
\hline
\end{tabular}

Table 1: Manual versus automatic registration. $\varpi$ is the warping index, $E$ is the average square difference. 'ideal' stands for the projection of the true transformation onto the warp space. This summarizes forty experiments.

techniques," Journal of Computer Assisted Tomography, vol. 21, no. 4, 1997.

[3] G. Christensen, S. Joshi, and M. Miller, "Volumetric transformation of brain anatomy," IEEE Transactions on Medical Imaging, vol. 16, Dec. 1997.

[4] S. Kiebel, J. Ashburner, J. Poline, and K. Friston, "MRI and PET coregistration - a cross validation of statistical parametric mapping and automated image registration," Neuroimage, no. 5, 1997.

[5] K. Rohr, H. S. Stiehl, R. Sprengel, W. Beil, T. M. Buzug, J. Weese, and M. H. Kuhn, "Point-based elastic registration of medical image data using approximating thin-plate splines," in Visualization in Biomedical Computing (K. H. Höhne and R. Kikinis, eds.), pp. 297-306, Springer-Verlag, 1996.

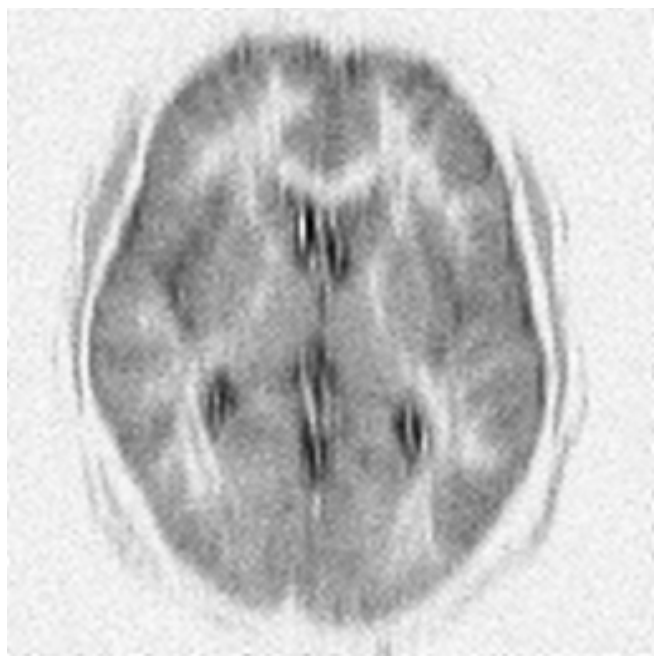

Figure 4: Artificial EPI image

[6] M. J. Gourlay, "xmorph." ftp://ftp.x.org/ contrib/graphics.

[7] F. Bookstein, Morphometric Tools for Landmark Data: Geometry and Biology. Cambridge University Press, 1997.

[8] J. Ahlberg, E. Nilson, and J. Walsh, The Theory of Splines and Their Applications. New York: Academic Press, 1967.

[9] I. Schoenberg, "Spline functions and the problem of graduation," Proc. Nat. Acad. Sci., vol. 52, pp. 947-950, 1964.

[10] M. Unser, A. Aldroubi, and M. Eden, "B-spline signal processing: Part I-theory," IEEE Transactions Signal Processing, vol. 41, pp. 821-832, Feb. 1993.

[11] M. Unser, A. Aldroubi, and M. Eden, "B-spline signal processing: Part II - efficient design and applications," IEEE Transactions Signal Processing, vol. 41, pp. 834-848, Feb. 1993.

[12] P. Thévenaz, U. E. Ruttimann, and M. Unser, "A pyramid approach to subpixel registration based on intensity," IEEE Transactions on Image Processing, vol. 7, pp. 1-15, Jan. 1998. 


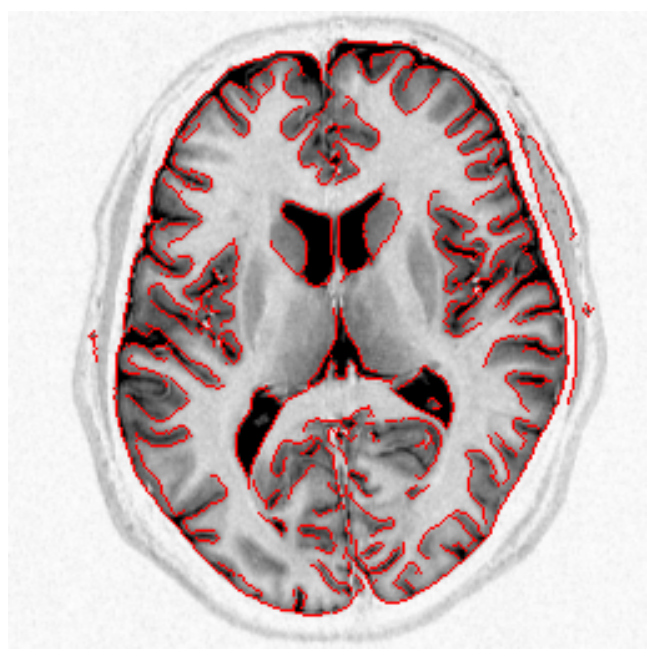

Figure 5: Anatomical picture

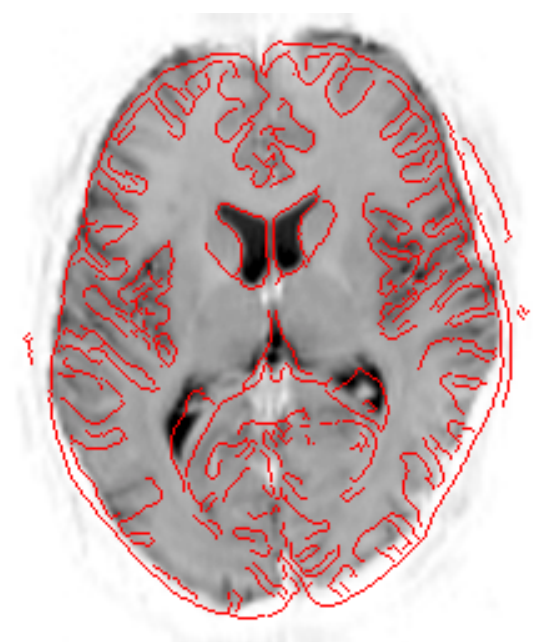

Figure 6: Original EPI

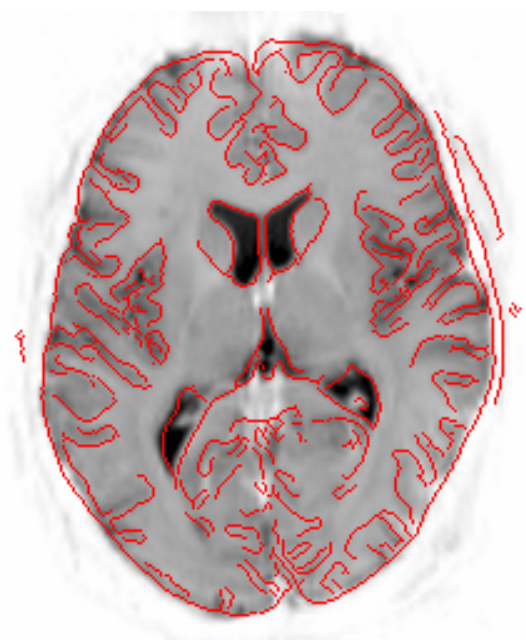

Figure 7: Automatic registration

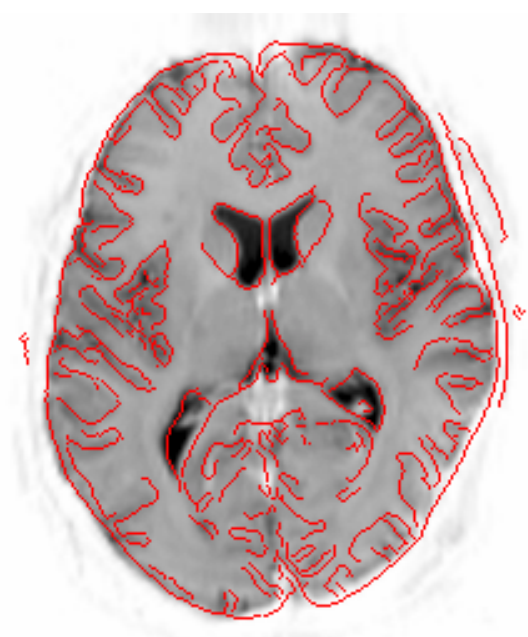

Figure 8: Manual registration 